\title{
Belgeo
}

Revue belge de géographie

$4 \mid 2016$

Sustainability of rural systems: balancing heritage and innovation

\section{Du bon usage du bocage : la haie bocagère au cœur des enjeux de développement durable}

The good use of the bocage system at the heart of sustainable development issues

\section{Mathieu Douet et Frédérick Lemarchand}

\section{OpenEdition}

\section{Journals}

Édition électronique

URL : http://journals.openedition.org/belgeo/19436

DOI : $10.4000 /$ belgeo.19436

ISSN : 2294-9135

Éditeur :

National Committee of Geography of Belgium, Société Royale Belge de Géographie

Référence électronique

Mathieu Douet et Frédérick Lemarchand, « Du bon usage du bocage : la haie bocagère au cœur des enjeux de développement durable », Belgeo [En ligne], 4 | 2016, mis en ligne le 31 décembre 2016, consulté le 10 décembre 2020. URL : http://journals.openedition.org/belgeo/19436 ; DOI : https:// doi.org/10.4000/belgeo.19436

Ce document a été généré automatiquement le 10 décembre 2020.

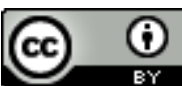

Belgeo est mis à disposition selon les termes de la licence Creative Commons Attribution 4.0 International. 


\title{
Du bon usage du bocage : la haie bocagère au cœur des enjeux de développement durable
}

The good use of the bocage system at the heart of sustainable development issues

\author{
Mathieu Douet et Frédérick Lemarchand
}

\section{Introduction}

Le système bocager, qui représente $56000 \mathrm{~km}$ de haies arbustives dans le département de la Manche, est un paysage à caractère anthropique qui est devenu un "écosystème cultivé " complexe et favorable au développement de la faune et de la flore (Larrère, Larrère, 1997). Cette forêt linéaire est donc née d'un imaginaire social-historique particulier, et non d'un hasard, démontrant que les sociétés humaines sont capables de transformer leur territoire sans remettre en cause leurs propres conditions de vie (Diamond, 2005). Biodiversité et enjeu climatique sont les clés de voûte des problèmes qui se posent à l'ensemble des sociétés humaines aujourd'hui, et des solutions durables à apporter (Bourg, Papaux, 2010). Face à ces grands enjeux environnementaux, les services de l'Etat ont lancé en 2012 une étude à l'échelle de la Normandie afin d'identifier six territoires cohérents, du point de vue de leur vulnérabilité aux effets du changement climatique, dans le but de réfléchir à la définition d'actions spécifiques en vue d'une meilleure adaptation de ceux-ci aux épisodes de sécheresse, de canicule ou tout autres évènements climatiques violents difficilement prévisibles (DATAR, 2013). Concernant le département de la Manche les analyses prédisent, pour les littoraux et la presqu'île du Cotentin, une baisse de la disponibilité en eaux et une aggravation du risque de submersion des côtes basses, avec les répercussions que nous connaissons sur la qualité de l'eau potable ou encore pour l'agriculture avec la perte de surface en herbe. Et, une transformation du bocage et des écosystèmes qui en dépendent avec des répercussions notables sur la baisse 
de la production de fourrage. C'est donc toute la productivité des exploitations normandes qui sera affectée en cas de non adaptation au changement climatique (Calame, 2008).

2 Face à des changements aussi radicaux et imprévisibles que sont les changements climatiques - et la déplétion des ressources fossiles -, les systèmes humains n'ont pas d'autre choix que de développer une capacité à réagir, une aptitude dite à la résilience (Cyrulnik, Duval, 2006). La "résilience locale » est donc la capacité d'un système humain, d'une société vivant dans un système écologique particulier, à conserver un état d'équilibre grâce à une capacité d'anticipation de ses acteurs vers un autre régime de comportement (Hopkins, 2008). Or, le bocage pourrait jouer de multiples fonctions d'amortisseur de crise écologique sur tous les plans grâce au maintien de la biodiversité, à la fourniture en énergie renouvelable et à la préservation des eaux de surface tout en contribuant à l'économie du territoire par le maintien de l'emploi agricole (Alphandéry, Dupont, 1985). C'est donc dans cette perspective que nous avons mené notre recherche sur les haies bocagère afin de comprendre et analyser les logiques socio-économiques qui sous-tendent l'organisation du système bocager tout en évaluant ses potentialités de valorisation agro-écologiques, socio-économiques, et notamment par l'intermédiaire de la création d'une filière bois-énergie. Nous avons donc choisi d'aborder cette recherche à travers une approche systémique et pluridisciplinaire - en menant une cinquantaine d'entretien ouverts auprès d'acteurs ressources et de terrain dans le département de la Manche, de l'Orne et du Calvados ${ }^{1}$-, tant pour saisir la complexité du point de vue du sens et de la transversalité de tous les enjeux, que pour être capable d'aborder simultanément les aspects économiques, sociaux et environnementaux (De Rosnay, 1975).

\section{Modernisation agricole et système bocager}

\section{Haie et compétitivité des modèles de production}

3 Après une période active de remembrement entre les années 1960 et 1980, en lien avec l'intensification et la mécanisation des pratiques agricoles (Mendras, 1975), on constate encore que l'une des causes premières de l'arrachage des haies est la recherche de compétitivité dans les systèmes de production agricoles (Prével, 2007). Aujourd'hui encore, la fin des quotas laitiers peut conduire à la concentration des structures d'exploitation, à leur agrandissement et donc à une potentielle perte de densité de bocage. La modification des choix de production de certains exploitants, qui sortent de l'élevage pour développer les systèmes de grandes cultures comme les céréales ou les oléagineux par exemple, pose problème pour le maintien des haies bocagères qui sont considérées comme une contrainte technique à leur modèle de production (Deléage, 2013). Un technicien de la Chambre d'agriculture de la Manche constate que « des décisions radicales [sont prises] chez certains agriculteurs qui passent de l'élevage laitier à la production de céréales. Les bocages denses disparaissent et il y a des agrandissements ». En 1964 on estimait par exemple que la Manche possédait $120000 \mathrm{~km}$ de haies (Brunet, Girardin, 2004). Dans le même temps, ces logiques productives accélèrent la baisse du nombre d'agriculteurs puisque dans le département de la Manche le recensement agricole met en lumière que le nombre total des exploitations est passé de 18242 exploitations en 2000 à 11328 exploitations en 2010. La population agricole est donc passée de 30881 actifs permanents à 20338 actifs permanents, sur cette même période, ce qui a des répercussions sur le 
système bocager via les agrandissements de structure notamment (AGRESTE, 2011). Or, « ce paysage ne peut subsister s'il n'y a pas assez d'agriculteurs pour l'exploiter et l'entretenir. [...] Les petits exploitants jouent un rôle déterminant dans l'entretien du bocage » (Alphandery, Dupont, 1985). Ainsi, si la diminution du nombre d'agriculteurs dans la Manche se poursuit les conditions de valorisation énergétique du bois-énergie vont se modifier et ne pourront potentiellement plus être réunies pour autoriser sa gestion au niveau des intercommunalités. Ainsi, dégradation du bocage et baisse du nombre d'agriculteur sont des éléments qui accélèrent la restructuration foncière (Alphandéry, Bitoun, Dupont, 1989).

Figure 1. Remembrement agricole : parcelle en blé de 20 ha (Sud-Manche).
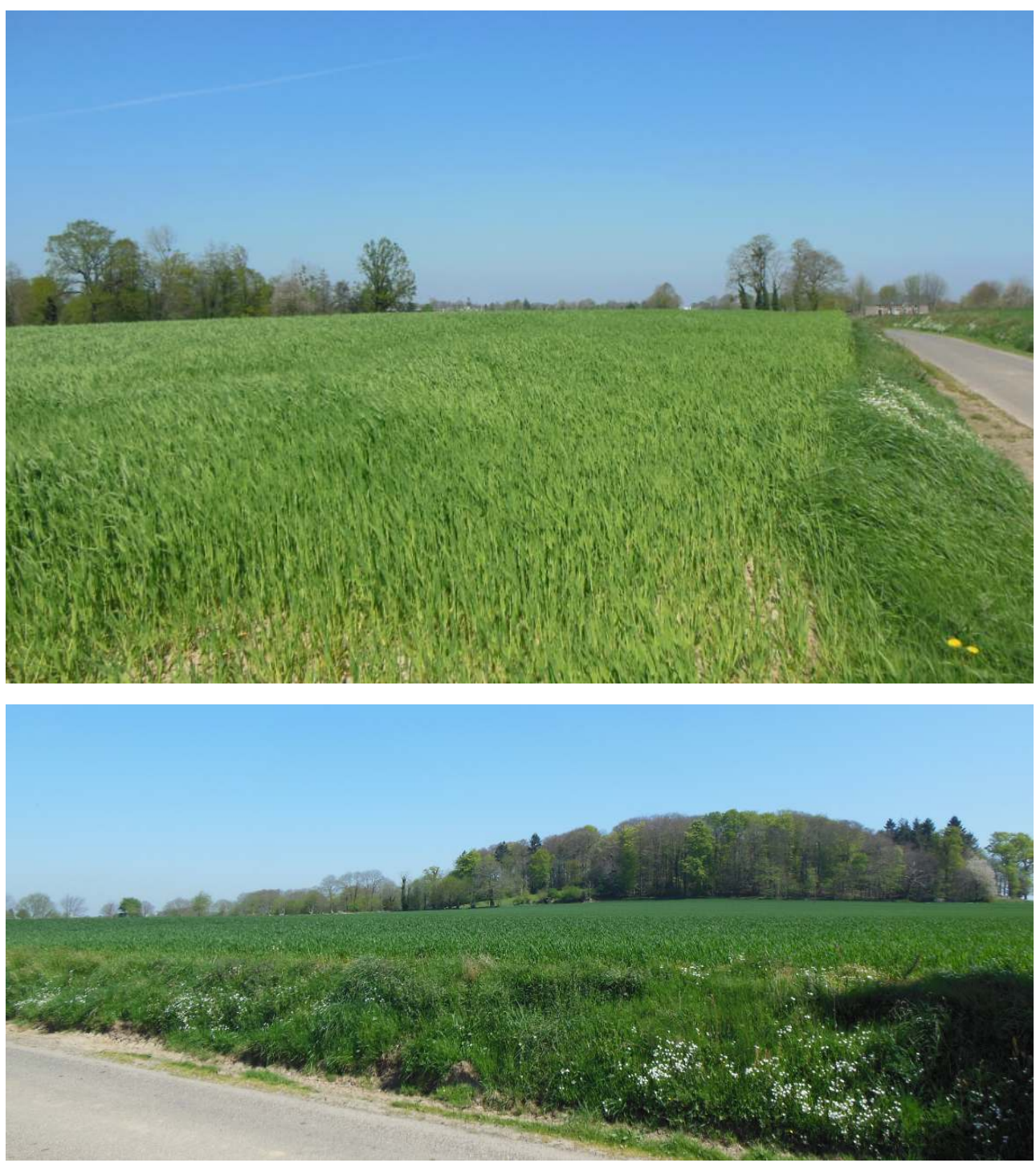

Source : Mathieu DOUET, Frédérick LEMARCHAND (2015)

\section{Appréhension et gestion différenciée du bocage}

Nous avons également pu remarquer que le manque de chiffres clairs sur le linéaire de haies participe à une construction «cornucopienne ${ }^{2}$ » de la ressource puisqu'il n'est pas 
rare d'obtenir des chiffres très différents concernant leur quantité en fonctions des acteurs interrogés. Cette omniprésence de la forêt linaire pourrait paradoxalement donner l'impression que la nature est inépuisable et inaltérable, exploitable à l'infini (Rist, 2001). Ainsi, entre défenseurs de l'environnement ou acteurs productivistes du monde agricole, les chiffres et les représentations de la ressource sont très différents. Les plus écologistes avancent des chiffres de $30000 \mathrm{~km}$ de haies, afin d'insister sur le caractère fragile de la ressource, alors que d'autres acteurs avancent le chiffre de 80000 $\mathrm{km}$ de haies (CG 50, 2009). Quant aux services déconcentrés de l'Etat, ils affirment, dans une position médiane, que la Manche possède bien $56000 \mathrm{~km}$ de haies mais que $20 \%$ ne sont que des talus enherbés. Cela ne participe donc pas à la construction d'une vision commune des potentialités de développement d'une filière bois-énergie, vision nécessaire à tout projet partagé de gestion de la ressource. Nous comprenons donc que le rapport à la haie reste fondamentalement complexe pour chaque individu et que nous ne pouvons réduire les agriculteurs à un seul type qui ne s'intéresseraient pas aux haies car elle ne leur rapporte rien économiquement (Castoriadis, 1975).

5 En effet, certains retiennent que la haie à des fonctions de protection des élevages par exemple, alors que d'autres vont principalement retenir la fonction paysagère (Godelier, 1984), surtout si leur exploitation comporte une fonction d'accueil (agrotourisme). La synthèse de ces visions plurielles nous conduit à identifier au moins cinq fonctions principales de la haie bocagère : agronomique et bois-énergie (économique), paysagère et patrimoniale (social), réservoir de biodiversité, anti-érosion et hydrique (environnementale). Cependant, dans cette approche par les entrées, nous comprenons que pour certains l'entretien et la préservation des haies ne soit pas un objectif prioritaire (Mazoyer, Roudart, 1997). Selon un technicien de la Chambre d'agriculture de la Manche « il y a une forte densité de haies sur le territoire manchois mais on constate que les proportions ne sont pas les mêmes entre le nord de la Manche et le sud-manche ». Dans certaines parties du territoire le linéaire de haies est de moins de $50 \mathrm{~m} /$ ha alors que dans d'autres parties on relève des densités de plus de $150 \mathrm{~m} / \mathrm{ha}$. L'aspect du bocage peut ainsi fortement varier d'une localité à une autre au point que certains parlent de «systèmes bocagers " au pluriel. Ces comportements distincts face à la ressource bocagère participent donc à la création d'un paysage bocager hétérogène et à la mise en lumière de comportements différents face à la ressource. Il apparaît ainsi que la difficulté de préservation du bocage serait en partie issue de la difficulté des acteurs économiques et agricoles à observer simultanément les grandes fonctions et rôles des haies bocagères sans les séparer (économiques, sociales et environnementales). Au final nous pouvons classer les agriculteurs en trois catégories:

- Ceux qui entretiennent la haie et qui en font une ressource ;

- Ceux qui l'ignorent et qui la laisse ;

- Ceux qui cherchent par tous les moyens à s'en débarrasser et/ou à en tirer un profit économique immédiat.

6 Cette typologie nous renseigne sur les différentes politiques qui pourraient être mises en œuvre à l'égard des exploitants agricoles selon le type de relation qu'ils entretiennent avec le bocage. Dans le premier cas il serait nécessaire de poursuivre et de renforcer l'accompagnement des producteurs-utilisateurs du bocage. Dans le second cas, les agriculteurs pourraient majoritairement déléguer leurs chantiers d'entretiens à des CUMA (Coopératives d'utilisation de matériel agricole) ou à travers d'autres types d'entreprises (privées, insertion par l'économique). Enfin, concernant le troisième cas, il 
est impératif qu'une filière d'entretien-valorisation se structure localement avec des acteurs économiques identifiés, car, sans organisation particulière, les exploitants feront intervenir n'importe quelle société privée d'abattage leur proposant des chantiers clés en main comme nous pouvons déjà le constater. On peut comprendre que la rapidité d'exécution des travaux de coupe sans Plan de gestion des haies (PGH) peut facilement séduire les agriculteurs voulant se débarrasser rapidement de la ressource bocagère, mais au détriment de toute logique de durabilité.

Figure 2. Avant et après arrachage massif de haies dans la commune de Lonlay-l'Abbaye (61).
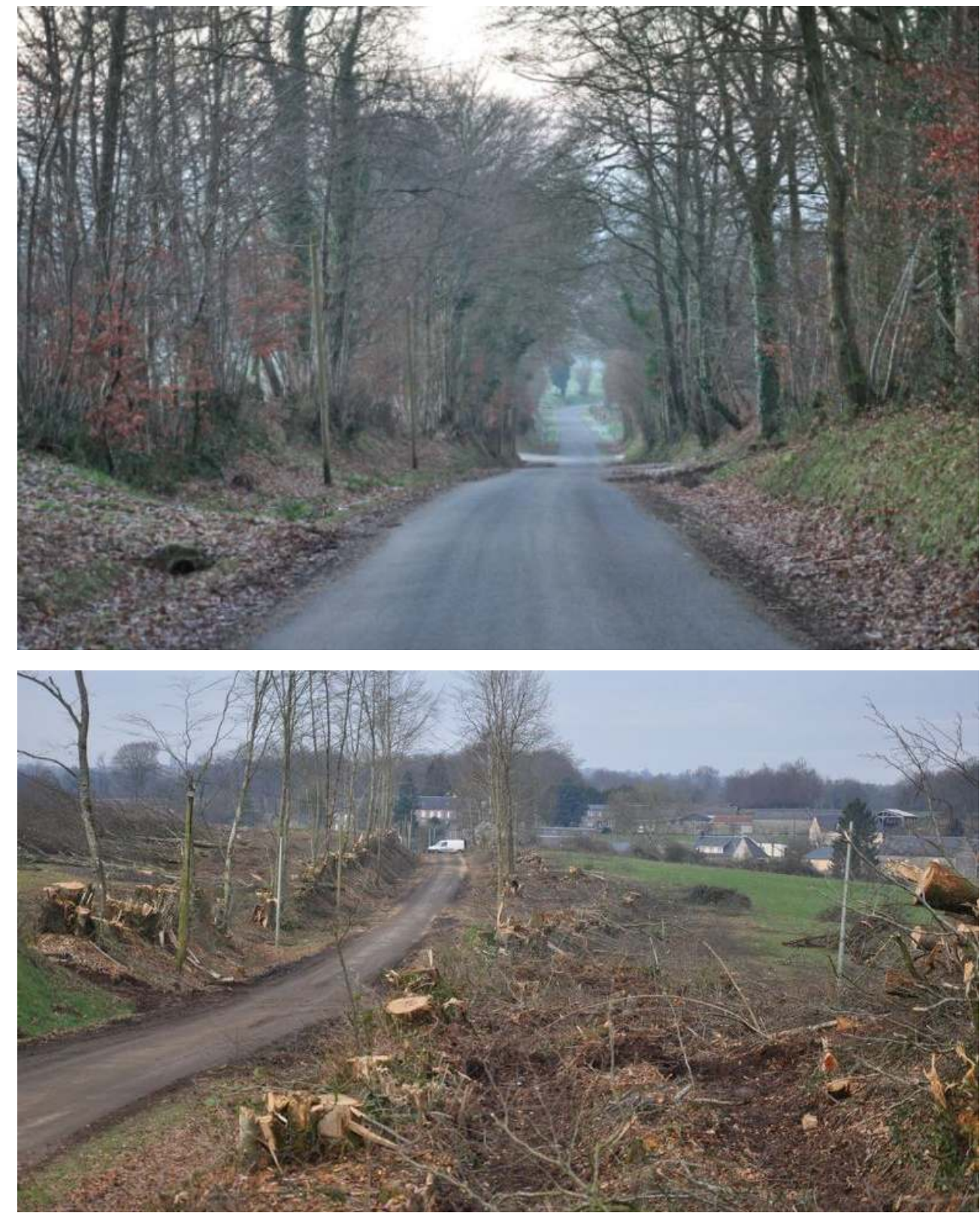

Source : Laurent NEVOUX (2015) 


\section{Une multiplicité de politiques publiques à inventer?}

7 On constate que les entrées sont multiples et que tous les agriculteurs n'entendent pas embrasser les mêmes fonctions et rôles pour la préservation et/ou la mise en valeur des haies sur leur exploitation (production, culturelles ou patrimoniales, régulation), et encore moins toutes en même temps. Les politiques publiques devraient s'inspirer de cette multiplicité afin de moins sectorialiser leurs actions en fléchant leurs aides. En effet, la complexité des questions d'ordre écologique appelle bien souvent des réponses régaliennes par l'intermédiaire de politiques sectorialisées (Pécaud, 2005). Ces réponses tendent souvent paradoxalement à masquer la cohérence globale et territoriale d'une politique volontariste en faveur du bocage que le Département pourrait mettre en œuvre. Selon certains acteurs et techniciens de la filière agricole, les politiques publiques sont donc pensées « sans aucune véritable concertation avec les acteurs de terrain » ce qui engendre des problématiques comme celles que la Manche a connu avec l'anticipation de la PAC en 2015 et qui a conduit à des arrachages massifs de haies par des agriculteurs qui estimaient que le législateur viendrait bientôt toucher " jusqu' à leur dernier espace de liberté » sur leur exploitation. En effet, l'exploitation du bois ainsi que les coupes à blanc sont autorisées, comme le recépage. La destruction d'une haie est également possible sur justifications jointe à la Déclaration préalable auprès de la DDTM : création d'un accès, agrandissement d'un bâtiment, sur décision administrative, etc. En revanche pour l'abatage des arbres de hauts-jets l'accord du propriétaire est indispensable. Tous les propriétaires n'ont pas le même rapport aux haies mais d'une manière générale ce sont eux qui sont le plus attachés à leur maintien puisqu'ils les qualifient de « capital sur pied » (Baudry, 2009).

Ainsi, selon des élus représentant du monde agricole conventionnel, « il faut rédiger une loi sur le bocage mais celle-ci aura du sens et pourra être appliquée si et seulement si elle est rédigée par des agriculteurs qui connaissent les contraintes de leur métier». Toutefois, certains acteurs du monde agricole engagés dans une démarche de développement durable, s'interrogent sur la possible instrumentalisation des agriculteurs par les représentants syndicaux qui auraient «données des consignes d'abatage à suivre». Par exemple, la Loi d'avenir pour l'agriculture, l'alimentation et la forêt (LAAAF) du 13 octobre 2014, encourage la réduction des intrants agricoles et à la mise en œuvre d'une agriculture agro-écologique où la haie aurait un réel rôle à jouer en tant que partie intégrante de l'agro-système et en particulier sur la qualité des rendements des cultures (Soltner, 1973). De fait, cet arrachage réalisé par anticipation de la nouvelle PAC est la matérialisation d'une lutte symbolique contre toute forme de «verdissement» de la PAC et des pratiques agricoles sur le territoire français (Bess, 2003). La conception de l'écologie vécue comme frein qu'ont développé les agriculteurs conventionnels est un point essentiel sur lequel les forces de révisions de la politique agricole devront travailler (Martin, 2011). Ces arrachages de haies sont ainsi l'expression d'un mouvement social contestataire porté par une frange de la profession agricole qui entend pérenniser son modèle technique de production intensif (Méda, 1999). Selon certains acteurs du monde conventionnel, il faut "cesser l'écologie punitive " car les normes environnementales actuelles "étoufferaient " les agriculteurs et sont donc vues comme coresponsables, avec le manque de compétitivité des exploitations françaises, de la crise agricole actuelle. Le monde agricole se sent pris entre le marteau de la complexité administrative des déclarations et l'enclume de la sanctuarisation des haies. Or, pour les acteurs économiques et associatifs de la filière bois-énergie, redonner une fonction économique à la haie est l'un des 
objectifs prioritaires d'une politique efficace de préservation de la ressource et d'une territorialisation économique (Magnaghi, 2000). Pour le monde agricole, il est effectivement important de mettre en avant la fonction économique afin de valoriser la haie puisque l'existence d'un débouché est fondamentale pour solliciter leur intérêt. On observe ainsi qu'il devient nécessaire d'engager un dialogue entre les différentes partiesprenantes, dialogue que certaines structurations juridiques rendent possible telles les Sociétés coopératives d'intérêt collectif (SCIC).

\section{La structuration d'une filière bois-énergie : potentialités et limites}

\section{Enjeu du maintien des Plans de plantation et de gestion des haies}

9 L'échelle locale est souvent décrite par les acteurs comme une bonne échelle pour être en mesure de "contrôler les bonnes pratiques et de démarcher le monde agricole et les élus locaux" (notamment dans le cadre des PLUi). De fait, la filière réclame plus d'animateurs sur les territoires afin d'être capable d'avoir une véritable démarche proactive vers les agriculteurs, et notamment les moins sensibilisés à la mise en œuvre de PGH; peu d'agriculteurs font la démarche d'aller vers la Chambre d'agriculture pour élaborer des PGH au sein de leur exploitation. Or, selon les acteurs de la filière agricole inscrits dans les démarches de valorisation de leurs bois de haies, il est essentiel de mettre en place des PGH car ceux-ci vont directement indiquer aux agriculteurs le réalisant le «tarif de cubage » et la rente potentielle de leur linéaire de haie. Le PGH peut donc permettre de sensibiliser directement la profession agricole aux enjeux de la préservation/valorisation des haies bocagères via le volet économique. En outre, il constitue une étape indispensable pour sécuriser dans le temps l'approvisionnement des chaufferies collectives en bois-énergie et permet aux agriculteurs de mieux appréhender les liens à retisser entre les acteurs du monde agricole et les collectivités locales dépendantes de leur production. Aussi, afin de pallier le déficit de demande de PGH, certains acteurs du département estiment que les Plans de plantation sont essentiels pour «capter " les agriculteurs et les sensibiliser dès l'amont de la filière. Ces replantations participent à pallier la baisse de productivité en bois du système bocager qui semble avérée dans le cadre d'un non remplacement régulier ou d'un mauvais entretien. Dans ce cas précis, certains techniciens estiment que l'un des aspects le plus visible de cette dégradation est la raréfaction des arbres de haut-jet.

10 Cependant, les agriculteurs avancent principalement trois contraintes concernant la préservation/valorisation de leurs haies : le manque de temps, la pénibilité du travail, le manque de marge alors qu'ils en supportent la totalité des charges. La volonté des acteurs associatifs de la filière bois-énergie est donc de faire passer l'entretien des haies du registre de la contrainte à celui d'une opportunité. Selon la SCIC «Bois bocage énergie » dans le département de l'Orne, leur activité professionnelle comprend trois fonctions principales destinées à dépasser ces problématiques :

- Acheter du bois vert broyé auprès des producteurs et le revendre en sec auprès de ses clients (après séchage sur plateforme) ;

- Elaborer des études de faisabilité ou d'assistance de maîtrise d'ouvrage pour des projets de chaufferies collectives ; 
- Démarcher les producteurs pour élaborer des PGH pour s'assurer que ceux-ci ont bien la ressource et qu'ils ne puissent pas dans leurs réserves.

11 A cette fin, il est important de mettre en œuvre une dynamique locale liant agriculteurs, collectivités et citoyens. Ainsi, bien que certains acteurs du département ou de la filière se posent la question de savoir si l'on doit continuer à soutenir autant la structuration de la filière bois-énergie par des aides aux boisements et aux postes d'animateurs locaux, il semble que ces efforts soient nécessaires pour continuer de participer à l'amorçage d'une filière bois-énergie locale (Association Haiecobois, 2014).

\section{Filière industrielle versus filière locale}

12 Selon certains élus et acteurs associatifs de la filière bois-énergie d'origine bocagère, la mise en œuvre d'une filière de type industriel engendre des problèmes de plusieurs ordres. Elle freine notamment le développement et la structuration d'une filière plus conforme au projet des élus locaux et des agriculteurs. De fait, la filière industrielle semble endiguer le développement en aval de la filière locale alors que celle-ci est une étape déterminante pour notamment aider les agriculteurs locaux à mieux percevoir l'intérêt de la préservation/valorisation de leurs haies. Nous comprenons alors que les deux filières, même si elles doivent s'inscrire dans une certaine complémentarité sur le papier, n'ont pas la même finalité. L'une vise un développement territorialisé qui cherche à entretenir le bocage en valorisant une ressource locale au profit des agriculteurs, des particuliers, des collectivités, tout en conduisant à une réduction des GES. L'autre cherche à maximiser l'utilisation de plaquettes forestières, ou de déchets de l'industrie du bois (scierie), et non spécifiquement à dépendre d'un approvisionnement local ou de structurer une filière locale sur le territoire manchois. Certains projets de grosses chaudières participent donc directement à la structuration d'une filière industrielle qui importe du bois forestier, même si celui-ci doit venir de l'étranger. Effectivement, l'approvisionnement en bois, c'est-à-dire le temps long écologique du bois, ne correspond pas au temps réel de l'économie ce qui pousse inéluctablement à l'augmentation de la distance d'approvisionnement pour les chaufferies industrielles (Clarke, Jo Sader, 2014). De fait, l'approvisionnement de l'étranger vient pallier ce manque et on constate que les grands importateurs-fournisseurs du bois se situent désormais à proximité des ports tels que Cherbourg, Rouen ou le Havre et importent du bois venant du Venezuela, d'Amérique du Nord ou de la Mer Baltique. Les chaufferies industrielles posent donc de nombreuses questions à la filière locale et doivent nous interroger sur les limites territoriales du développement d'une filière bois-énergie qui pourrait contribuer à la construction d'un modèle énergétique qui déplace la charge environnementale de son développement l'empreinte écologique (Bouteau, Gondran, 2009) - sur les territoires voisins qui peuvent quant à eux exploiter cette ressource de façon totalement non durable.

En outre, si la superficie de forêt en France progresse de $+0,6 \%$ par an depuis les années 1980 pour atteindre actuellement 16,5 millions d'hectares, celle du bocage continue de régresser d'au moins $1800 \mathrm{~km}$ de haie par an selon les acteurs de la filière dans le département de la Manche (IGN, 2013). A ce rythme de disparition, et sans replantation systématique de haies dans le département, c'est l'équivalent de $63000 \mathrm{~km}$ de haies qui auront disparu à l'horizon 2050, voire $153000 \mathrm{~km}$ de haies en moins d'ici la fin du siècle, soit de 1 à 2,7 fois le linéaire de haies actuel dans la Manche. De surcroît, le taux de boisement de la France est de $30 \%$ mais varie très fortement puisqu'il est de $67 \%$ en 
Corse-du-Sud par exemple pour seulement $5 \%$ dans la Manche. Ainsi, la filière industrielle ne peut donc pas à elle seule porter la préservation/valorisation des boisbocagers et présente au final un intérêt limité pour les spécificités du territoire manchois et sa ressource face aux atouts d'une économie territorialisée (Caillé, 1989). Aussi, par rapport au marché global du bois déchiqueté, le bois d'origine bocagère doit rester limité aux petites chaudières puisque celles-ci ont des contraintes techniques de fonctionnement et ne peuvent pas utiliser un bois déchiqueté avec un taux d'humidité élevé $(+25 \%)$ ou un déchiquetage grossier par exemple ${ }^{3}$. C'est donc par ce type de constat que les acteurs de la filière bois-énergie d'origine bocagère estiment que la filière locale est plus pertinente, en conduisant à freiner la régression du système bocager dans la Manche, mais également en valorisant au mieux cette ressource. Ainsi, sans tenter de démontrer que les chaudières industrielles n'ont pas d'intérêt, puisqu'elles demeurent dans le cercle vertueux de l'énergie renouvelable, nous pouvons néanmoins constater que ce type d'équipement freine tous les porteurs de projets locaux puisque les grosses chaufferies captent un gisement qui aurait pu alimenter de petites chaufferies - parfois encore inexistantes sur le territoire -, ce qui induit des effets à la fois de concurrence (qui nuisent à la complémentarité) et d'importation massive de bois remettant en cause la notion même d'autonomie énergétique des territoires. Toutefois, dans une acception purement économique, la dispersion de la production des bois bocagers - forêt linéaire -, la mécanisation croissante des chantiers et le temps de séchage plus long et précis de la filière bocagère pèsent sur les coûts de production, ce qui freine aussi intrinsèquement le développement de la filière plus locale et nécessite, là encore, une vision plus territoriale que strictement économique du problème ainsi que des analyses à moyen et long terme.

\section{Bois-énergie : atout pour penser le développement durable des territoires}

\section{Coût de mobilisation de la ressource ou projet de territoire}

Ces constats nous indiquent que ces questions sont d'abord une affaire de choix, donc d'orientation politique, et non purement de choix techniques (Rumpala, 2015). Par exemple, faut-il installer 1000 chaudières collectives de 100 Kilowatt $(\mathrm{kW})$ ou une seule chaudière comme à Rennes de plus 30 Mégawatt (MW) qui réclame 130000 tonnes de bois par an, c'est-à-dire 20 semi-remorques par jour? Ce sont, au final, les choix énergétiques qui seront élaborés par les acteurs politiques et économiques de la filière qui vont structurer à court ou moyen termes le maintien des grands équilibres écologiques et la qualité des conditions de vie des individus sur un territoire (Partant, 1982). Selon un acteur associatif de la filière bois-énergie d'origine bocagère dans la Manche, «le bois présente un avantage compétitif considérable pour les collectivités locales par rapport aux autres sources d'énergie conventionnelles». Par exemple, l'association "Haiecobois» livre la chaufferie locale de la commune de Saint-Samson-de-Bonfossé dans la CDC de Canisy dans le centre-Manche. La chaudière de $85 \mathrm{~kW}$ alimente un réseau de chaleur de 160 mètres de long pour 10 logements HML destinés à des personnes âgées. La consommation des logements d'en moyenne $66 \mathrm{~m}^{2}$ est de 35 tonnes de bois-sec par an. Elle est l'équivalent de la valorisation de 500 mètres de haies/an, ou de $8 \mathrm{~km}$ de haies entretenus en rotation sur 15 ans, au lieu de 11200 litres de fioul (31 tep ou 96T de $\mathrm{CO}_{2}$ ). On constate de fait qu'il est possible pour une collectivité de diminuer son empreinte énergétique tout en faisant des 
économies financières. Cependant, on constate que le prix du bois d'origine bocagère vendu par des associations s'avère «supérieur au prix du bois-déchets de $2000 €$ à $2500 €$ par an » selon Haiecobois. En effet, le bois bocager dépend du «prix d'entretien » et du prix de «mobilisation de la ressource» ce qui impacte inévitablement le prix d'achat qui est, du coup, jugé encore trop cher par certaines collectivités ${ }^{4}$. Toutes les collectivités locales ayant mis en place des chaudières bois ne s'approvisionnent donc pas forcément en bois local alors qu'il serait plus pertinent de mobiliser la ressource "dans un rayon d'une quarantaine de kilomètres au maximum » selon les acteurs de la filière bocagère consultés.

Dans le même temps, les acteurs de la filière bois-énergie dans l'Orne affirment qu'il y a encore des problématiques économiques qui freinent la structuration d'une filière boisénergie locale en amont de la filière, tel que le coût du kWh encore vendu trop bas pour définitivement intéresser tous les agriculteurs d'un territoire donné et concernés par la mise en œuvre d'une chaudière bois collective. Ainsi, un technicien de la filière boisénergie d'origine bocagère explique que «le $k W h$ est actuellement à $0,03 €$ ce qui donne un prix approximatif de $100 €$ la tonne de bois-déchiqueté, soit 65 à $70 €$ la tonne achetée à l'agriculteur. Or, si le $k W h$ pouvait passer à $0,05 €$, alors les collectivités achèteraient la tonne approximativement à $150 €$ ce qui serait beaucoup plus favorable à la structuration d'une filière bois-énergie locale ». Ainsi, il faut comprendre que la sollicitation de ces deux ressources - bois-bocage ou boisforêt - ne relève pas du même projet de territoire. L'objectif de structurer une filière boisénergie locale est en effet d'établir un nouveau mode de fonctionnement soutenable de la production-distribution-consommation d'énergie dans un esprit de coopération avec les acteurs économiques locaux tels que les agriculteurs (Laville, 2007). La préservation du bocage et sa valorisation par une filière locale repose ainsi sur un mouvement faisant passer les collectivités locales d'une logique d'économie de marché (hétéronome) à une logique d'économie de territoire (autonome). De fait, la lente structuration d'une filière bois-énergie locale tend à mettre en exergue la difficulté pour certaines collectivités locales de dépasser les règles actuelles de l'économie mondialisée - qui empêchent la soutenabilité des territoires locaux - en privilégiant la compétitivité plutôt que la coopération (Cattani, Laville, 2006). Le développement durable d'un territoire ne doit donc pas reposer sur un seul et unique pilier économique, hors de réalités globales, et prendre en considération une logique d'investissement de plus long terme relevant de « valeurs » communes aux acteurs au profit de la qualité de son développement immédiat et de celui des générations futures.

\section{Offrir un service clé en main aux collectivités : du producteur à la chaudière}

Après notre enquête il semble qu'il existe des éléments clés pouvant participer activement à la création et à la structuration d'une filière bois-énergie locale rémunératrice pour l'agriculteur et économique pour la collectivité :

- Le pilotage de la filière par une Commission locale composés d'élus, d'agriculteurs, de membres d'une CUMA voire de citoyens : ce type de Commission peut par exemple émettre un avis sur les demandes de déclaration préalable pour l'arasement d'une haie avec l'appui d'un technicien bocage ;

- Le recrutement d'un technicien chargé de la conception, du suivi, de la gestion des plans de plantations puis des chantiers de coupe ou de déchiquetage : ils sont des passerelles entre 
exploitants et institutions publiques pour aider les agriculteurs à prendre connaissance des dispositifs d'aides par exemple ;

- Des opérations de plantations se déroulant sur le territoire d'une commune, ou d'une CDC (PLUi), sur une période assez longue pour un minimum d'impact en termes de densité des haies replantées: mettre en marche un programme de recomposition du bocage avec des associations travaillant dans l'insertion professionnelle par exemple ;

- L'appui des partenaires publics - Conseil Régional ou Départemental - à la plantation et à l'animation : comme par exemple la CDC de Saint-James (50) qui s'est dotée d'un technicien bocage grâce aux subventions allouée à $100 \%$ par l'Agence de l'eau Seine-Normandie et la Région Basse-Normandie.

17 Aussi, bien que nous constations de nombreux freins, il existe une volonté politique de porter la structuration d'une filière bois-énergie largement entendue par le milieu agricole. Cependant, la sphère politique a encore des difficultés méthodologiques pour travailler avec le milieu agricole et celles-ci sont communes puisque leurs modes de fonctionnement respectifs entrent régulièrement en confrontation. En effet, d'un point de vue du développement urbain par exemple, le différentiel de valeur entre le foncier agricole et le foncier devenu urbanisable est tel que la vocation productive et énergétique des territoires ruraux est beaucoup moins prise en compte. La rente foncière accélère donc certaines dynamiques territoriales et pérennise le clivage entre territoires ruraux et territoires urbains (Lévy, Emelianoff, 2011).

Figure 3. Plan de plantation et plateforme de séchage/stockage de plaquettes.

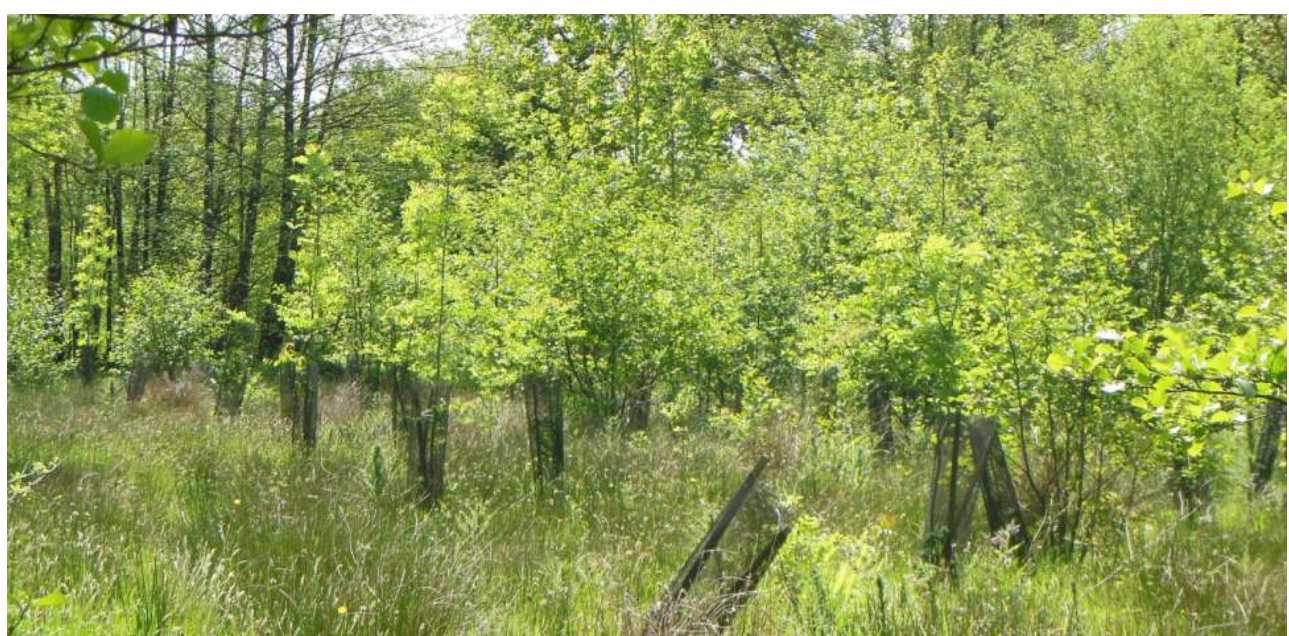




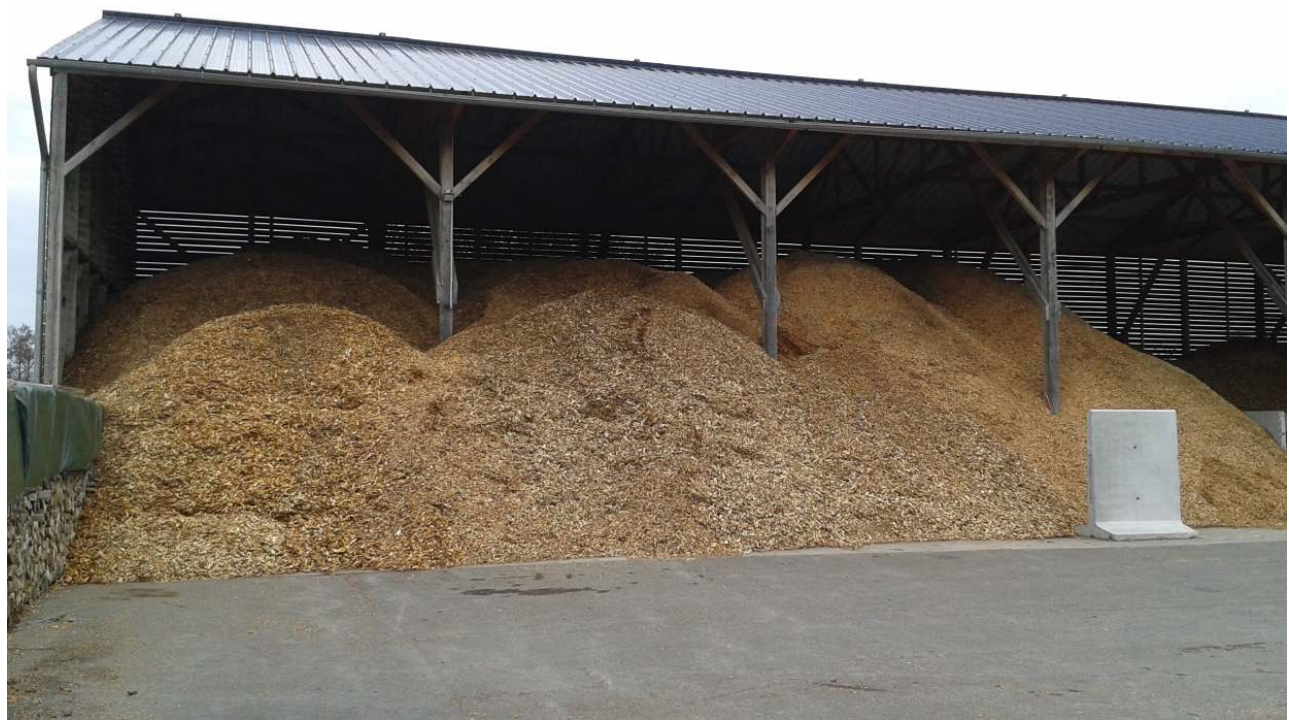

Source : Mathieu DOUET, Frédérick LEMARCHAND (2015).

Tout autant, un frein important à cette structuration provient du fait que les agriculteurs attendent que la sphère politique «prépare le terrain» en aval ce qui les pousse à l'attentisme quant au développement de ce secteur. En effet, le monde agricole a ses propres contraintes de production, spécifiques à tel ou tel mode d'exploitation, et n'entend pas non plus avoir vocation à se substituer aux élus quant à la mise en œuvre d'un développement durable local. De facto, si on synthétise l'ensemble des discours d'élus, des techniciens, des agriculteurs conventionnels ou non-conventionnels, créer le débouché pour les collectivités locales reviendrait notamment à passer par :

- Le recensement des projets de rénovation ou de construction de bâtiment publics sur un territoire par une collectivité : c'est donc la question du renouvellement des systèmes de chauffage qui doit permettre aux collectivités de réinterroger leurs dépenses énergétiques;

- La mise en place d'études comparatives bois-énergie/énergie-fossile : comme par exemple le SDEM propose aux collectivités locales ;

- Créer localement une plateforme de stockage et de séchage du bois, le mieux étant que plateforme de stockage et chaufferie constituent un seul et même équipement (sécher sur le lieu de consommation): les collectivités locales doivent prendre à leur charge les plateformes de séchage/stockage des plaquettes de bois afin que ce ne soit pas les agriculteurs qui supportent le poids économique de ces installations ;

- Sécuriser l'approvisionnement en gérant la ressource en favorisant à l'échelle des exploitations, ou d'un territoire, la remise en état des haies (plantation afin de renforcer la capacité de production des haies) et leur gestion durable (valorisation sans épuisement de la ressource).

\section{Conclusion}

19 La Manche est le premier département français pour son linéaire de haie et cette richesse patrimoniale lui confère une valeur environnementale supérieure à la moyenne lui permettant de mettre ses atouts au cœur 'un projet de «transition écologique » (Hopkins, 2008). Nous avons pu observer qu'il y a une réelle volonté politique pour porter la structuration d'une filière bois-énergie - matérialisée par des investissements 
(chaudières collectives, plateformes de stockage-séchage du bois) - et cette motivation a été entendue par le milieu agricole qui analyse de son côté les potentialités économiques de cette filière. Toutefois, le milieu agricole reste attentiste vis-à-vis de la sphère politique et délaisse encore trop à celle-ci la responsabilité de la structuration de la filière par la création de chaufferies collectives en aval. Cette lente structuration démontre ses difficultés à faire sens auprès de tous les acteurs sous l'acception de l'élaboration d'un projet commun de territoire puisque l'objectif de structurer une filière bois-énergie locale est bien d'établir un nouveau mode de fonctionnement soutenable de la productiondistribution-consommation d'énergie dans un esprit de coopération avec les acteurs économiques locaux. Penser une méthodologie de travail pour la concertation de ces acteurs n'implique donc pas de renforcer l'appareil réglementaire de planification mais de faire passer les acteurs d'un territoire de comportements relevant d'enjeux personnels de milieux à des enjeux collectifs de structure sociale (Wright-Mills, 1967). Le développement durable d'un territoire ne peut donc pas reposer sur un seul et unique prisme économique court-termiste et prendre en considération une logique d'investissement de plus long terme relevant de valeurs communes aux acteurs d'un territoire au profit de la qualité de son développement immédiat et de celui des générations futures. Cependant, et c'est peut-être là la principale limite à cette volonté de reterritorialisation économique, l'état de dégradation du tissu économique et social de certaines communes manchoises - perte d'emplois agricoles, fermetures d'écoles, déserts médicaux - endigue potentiellement toute forme d'initiative en matière de valorisation des ressources locales et de développement territorialisé.

\section{BIBLIOGRAPHIE}

AGRESTE (2011), Premiers résultats du recensement agricole 2010. Département de la Manche (50), AGRESTE Données, Basse-Normandie, $n^{\circ}$ 50, octobre 2011.

ALPHANDÉRY P., DUPONT Y. (1985), Développement local et société rurale dans l'isthme du Cotentin. Les aspects sociologiques de la valorisation énergétique de la biomasse, INRA, novembre 1985.

ALPHANDÉRY P., BITOUN P., DUPONT Y. (1989), Les champs du départ : une France rurale sans paysans ?, La Découverte.

ASSOCIATION HAIECOBOIS (2014), Rapport d'activité de l'exercice 2013-2014 et perspective de développement pour l'exercice 2014-2015, septembre 2014.

BOURG D., PAPAUX A. (dir.) (2010), Vers une société sobre et désirable, PUF.

BOUTEAU A., GONDRAN N. (2009), L'empreinte écologique, La Découverte.

CAILLÉ A. (2003 [1989]), Critique de la raison utilitaire. Manifeste du MAUSS, La Découverte.

CALAME M. (2008), La tourmente alimentaire. Pour une politique agricole mondiale, Charles Léopold Mayer.

CATTANI A.D., LAVILLE J.L. (dir.) (2006), Dictionnaire de l'autre économie, Gallimard. 
CAZENAVE M. (1996), Encyclopédie des symboles, La Pochothèque.

CONSEIL GÉNÉRAL DE LA MANCHE (2013), Ecrivons ensemble la Manche de demain! Démarche de prospective territoriale. Les enjeux - les futurs souhaitables - les scénarios, CG de la Manche.

CONSEIL GÉNÉRAL DE LA MANCHE (2009), Les différents patrimoines boisés dans la Manche, CG 50.

BAUDRY J. (2009), Trames Vertes, Programme de recherche " paysage et développement durable », INRA Sad-Paysage.

BESS M. (2011 [2003]), La France vert clair. Ecologie et modernité technologique 1960-2000, Champ Vallon,

BRUNET P., GIRARDIN P. (2004), Inventaire régional des paysages de Basse-Normandie, DREAL.

CASTORIADIS C. (1975), L'Institution imaginaire de la société, Seuil.

CLARKE B., JO SADER M. (2014), Centrale biomasse : la forêt française en danger ?, Actu-

Environnement.

CYRULNIK B., DUVAL P. (2006), Psychanalyse et Résilience, Odile Jacob.

DATAR (2013), L'adaptation aux effets du changement climatique en haute et Basse-Normandie. Analyse du climat futur en Haute et Basse-Normandie, SGAR de Haute et Basse-Normandie.

DELÉAGE E. (2013), Agricultures à l'épreuve de la modernisation, QUAE.

DE ROSNAY J. (1975), Le macroscope. Vers une vision globale, Seuil.

DIAMOND J. (2009 [2005]), Effondrement. Comment les sociétés décident de leur disparition ou de leur survie, Gallimard.

GODELIER M. (1984), L'idéel et le matériel, Fayard.

HOPKINS R. (2010 [2008]), Manuel de transition. De la dépendance au pétrole à la résilience locale, Ecosociété, Québec.

IGN, La superficie forestière en France métropolitaine, Institut national de l'information géographique et forestière, Campagnes 2009 à 2013.

LARRÈRE C., LARRÈRE R. (1997), Du bon usage de la nature. Pour une philosophie de l'environnement, Aubier.

LAVILLE J.L. (2007), L'économie solidaire. Une perspective internationale, Hachette.

LEVY A., EMELIANOFF C. (2011), « Quelles ville durable ?», Espaces et sociétés, nº 147.

MAGNAGHI A. (2003 [2000]), Le projet local, Liège, Pierre Mardaga.

MARTIN J.P. (2011), La Confédération paysanne aujourd'hui. Un syndicat face aux défis du 21ème siècle, L'Harmattan.

MAZOYER M., ROUDART L. (2002 [1997]), Histoire des agricultures du monde. Du néolithique à la crise contemporaine, Seuil.

MÉDA D. (2008 [1999]), Au-delà du PIB. Pour une autre mesure de la richesse, Flammarion.

MENDRAS H. (1995 [1975]), Les sociétés paysannes. Éléments pour une théorie de la paysannerie, Gallimard.

PARTANT F. (1997 [1982]), La fin du développement. Naissance d'une alternative ?, Babel.

PÉCAUD D. (2005), Risques et précautions. L'interminable rationalisation du social, La Dispute.

PRÉVEL M. (2007), L'usine à la campagne. Une ethnographie du productivisme agricole, L'Harmattan. 
RIST G. (2001), Le développement. Histoire d'une croyance occidentale, Presses de science Po.

RUMPALA Y. (2015), « Formes alternatives de production énergétiques et reconfigurations politiques. La sociologie des énergie alternatives comme étude des potentialités de réorganisation du collectif », in ZÉLEM M.C., BESLAY C., Sociologie de l'énergie. Gouvernance et pratiques sociales, CNRS Éditions.

SOLTNER D. (1995 [1973]), L'arbre et la haie : pour la production agricole, pour l'équilibre écologique, et le cadre de vie rurale, Collection sciences et techniques agricoles.

WRIGHT-MILLS C. (1997 [1967]), L'imagination sociologique, La Découverte.

\section{NOTES}

1. On compte des acteurs institutionnels (Conseil régional, Conseil départemental, élus locaux), des techniciens et des chargés de mission départementaux (bois, rivières, Parc naturels), des techniciens de la Chambre d'agriculture (bocage, polyculture, élevage), des représentants syndicaux du milieu agricole (Confédération paysanne, FNSEA), des agriculteurs, des associations environnementalistes, des techniciens et entrepreneurs de la filière bois-énergie, des universitaires.

2. L'esprit cornucopien est celui qui croit dans la corne d'abondance et par extension, possède une foi dans la science, le progrès et la technique. Elle est le symbole de richesses inépuisables offertes à l'homme (Cazenave, 1996).

3. Les bois humides sont moins performants et produisent moins de chaleur en brûlant - l'eau étant incombustible - puisque l'évaporation de l'eau, contenue dans le bois, va absorber de grandes quantités de chaleur et modifier les réactions chimiques de combustion, ce qui augmente systématiquement le taux de bois imbrûlé.

4. Environ $50 € \mathrm{TTC} / \mathrm{t}$ pour le bois issu de déchets ou de la transformation du bois et environ $80 €$ $\mathrm{TTC} / \mathrm{t}$ pour la plaquette forestière ou bocagère.

\section{RÉSUMÉS}

La présente proposition repose sur la réalisation d'une recherche pour le compte du Conseil départemental de la Manche portant sur les dynamiques de préservation/valorisation du système bocager manchois en lien avec une réflexion prospective plus globale engagée par cette collectivité territoriale. Dans le cadre d'une approche systémique et pluridisciplinaire, nous avons conduit une enquête d'un an - comptant une cinquantaine d'entretiens ouverts - afin de comprendre et analyser les logiques sociales qui sous-tendent l'organisation du système bocager actuel tout en évaluant ses potentialités de valorisation agro-écologiques, socio-économiques, et notamment par l'intermédiaire de la création d'une filière bois-énergie entrant dans le futur «mix énergétique ».

The following proposition stands on a research elaborated for the Conseil départemental de la Manche which is focusing on dynamics of preservation/valorization of the bocage system in the department of Manche, France. Besides, this research is intertwined with a broader reflection 
undertaken by the territorial collectivity. Within a systemic and multidisciplinary approach, we have conducted a one-year investigation - comprising around fifty open interviews - in order to understand and analyze the social logics behind the organization of today's bocage system while evaluating its agroecological and socioeconomical potentialities of valorization, particularly through the creation of a wood-energy sector as part of the future "energy mix".

INDEX

Mots-clés : services écosystémiques, bois-énergie, transition écologique, résilience locale Keywords : ecosystem services, wood-energy, ecological transition, local resilience

\section{AUTEURS}

MATHIEU DOUET

MRSH-CNRS - USR 3486, Université de Caen Normandie, douetmathieu@gmail.com

\section{FRÉDÉRICK LEMARCHAND}

MRSH-CNRS - USR 3486, Université de Caen Normandie, frédérick.lemarchand@unicaen.fr 